\title{
Relative aversiveness of subcutaneous shock and footshock in the rat ${ }^{1}$
}

BYRON A. CAMPBELL ${ }^{2}$ and WILLIAM H. MOORCROFT, ${ }^{3}$ PRINCETON UNIVERSITY, Princeton, New Jersey 08540

Relative aversiveness of subcutaneous shock and footshock was assessed by means of a spatial preference technique. Subcutaneous shock was less aversive than footshock by a factor of 10 , except at near lethal shock intensities, where receptor-cell hyperpolarization, electrocauterization, and/or muscular tetanization may have occurred.

Since the turn of the century psychologists have been searching for the ideal way to administer electric shock-induced pain to both primate and infraprimate Ss. In small mammals the technique of choice has been to apply shock to the feet through a stainless steel grid floor, with the pattern of shock varied constantly by some type of a scrambling circuit. The disadvantages of this type of circuit are obvious. The pattern and frequency of shock to the four extremities are constantly and unpredictably varying. Moreover, the proportion of time $\mathbf{S}$ receives shock depends upon the vagaries of the animal's bodily position and the characteristics of the grid scrambler employed.

For these and other reasons (cf. Campbell \& Masterson, 1969) psychologists have examined a variety of fixed-electrode techniques designed to eliminate variations in shock intensity and duration. Azrin and his associates (Hake \& Azrin, 1963; Hutchinson, Azrin, \& Hake, 1966) have described a method of delivering shock to the tail of a monkey, while Weiss (1967) reported the use of tail electrodes in rats. Kelly and Glusman (1964) and Appel (1965, cited in Weiss, 1967) developed subcutaneous methods of delivering shock, and Barfield and Sachs (1968) used safety pins as electrodes.

One major problem in using implanted fixed-electrode techniques is the difficulty of comparing the behavioral results from studies using subcutaneous stimulation with those using the more prevalent footshock procedures. That substantive differences in the painfulness produced by the two methods do exist is suggested by the unusually high aversion thresholds reported by Kelly and Glusman (1964) for subcutaneous shock.

\section{EXPERIMENT 1}

The purpose of this experiment was to compare the relative aversiveness of subcutaneous shock and footshock by means of a spatial preference technique. Rats were given a "choice" between various intensities of footshock and subcutaneous shock in order to determine equal aversion points (points of subjective equality) for a number of subcutaneous shock intensities.

\section{Method}

The behavioral method of this experiment incorporates the spatial preference task used in previous studies (e.g., Campbell \& Masterson, 1969). $S$ is placed in the preference cage and receives one stimulus when it is on the right side and another on the left, and he simply chooses the least aversive side. In the present experiment, one or two pairs of electrodes were implanted subcutaneously in albino rats, one pair near the neck and the other near the tail. When $S$ was on one side of the cage, shock was delivered through one pair of electrodes; on the other side, $\mathrm{S}$ received footshock via the grid floor of the cage. Relative aversiveness of the two shocks was assessed by relative preference for one side or the other.

Subjects. The Ss were Sprague-Dawley 275. to 350-g adult male rats obtained from the Charles River Breeding Laboratory, Wilmington, Mass.

Apparatus. The spatial preference cage was $14 \frac{1}{2}$ in. long, $7 \mathrm{in}$. wide, and $7 \mathrm{in}$. high. The sides and top were galvanized iron and the floor consisted of $3 / 32$-in. stainless steel grids mounted in a Plexiglas frame, spaced $1 / 2$ in. apart, center to center. The floor pivoted on a central axle, and a microswitch either opened or closed as the animal crossed from side to side, permitting control of shock, and recording of time spent on either side and the number of crossings.

The top of the cage had a slot $11 \frac{1}{2}$ in. long and $3 / 8 \mathrm{in}$. wide running the length of the cage. A Lehigh Valley Electronics 1373-4 slip-ring assembly was mounted 12 in. over the cage. The animal-to-swivel leads went from the slip-ring assembly to the animal through the slot in the cage top and were covered for the first 6 in. with a stiff jacket made of shrinkable tubing and dental cement to prevent gnawing.

Two constant-current ac shock sources with a $500-\mathrm{V}$ source voltage (Campbell \&
Masterson, 1969) were used; one delivered shock to the connector on the slip-ring assembly and then to the animal, and the other delivered shock to the grid floor. The subcutaneous shock was not continuous, but consisted of a train of 133-msec pulses separated by $67 \mathrm{msec}$ of no shock in Experiment 1. Preliminary work suggested that this procedure produces less physiological damage and disnuption than continuous shock. Shock applied to the feet was continuous, except that the pattern of shock applied to the grids was changed every $200 \mathrm{msec}$.

Electrode assemblies. The electrodes (Part No. 96-600-88) from the E.\& M. Instrument Co., Houston, Texas) consisted of a solid silver ring, $3 / 8 \mathrm{in}$. in diam, attached to a Teflon-coated stainless steel wire. The free end of this wire was cut (I and $1 \frac{1}{4}$ in. from the electrode for the anterior placements and $7-1 / 8 \mathrm{in}$. for the posterior placements), and then attached to a Poke Home contact (Amphenol Connector Division, Amphenol Corporation, 1830 S. 54th Ave., Chicago, Illinois 60650 , No. 220-pol). Two anterior and two posterior electrode assemblies were attached to a $2 \times 2$ plug made from pieces of Amphenol strip connectors (No. 221-1160) by means of Poke Home contacts. A thin solution of dental cement (L. D. Caulk Co., Milford, Delaware) was applied to the plug on the side trailing the wires to insure electrical insulation.

Surgical procedures. The Ss were anesthetized with Nembutal followed by chloral hydrate if nccessary (after Valenstein, 1961). Following surgery they were given Mikedmide (Parlam, Englewood, New Jersey) to speed revival. S was fixed in a stereotaxic head holder and the skin over the skull was incised and reflected. For each electrode placement, a $1 / 2$-in. incision was made in the skin, which terminated at another cut of like size but at a 90-deg angle to the first. These incisions were caudal and distal relative to the eventual electrode location. At this time a string was affixed to a hook in the end of a stainless steel rod (1/16 in. in diam) and directed under the skin from the skull incision to the electrode placement incision. The circular electrode was tied to the skull side of this string and then pulled under the skin to its eventual locus, trailing its attached stainless steel Teflon-coated wire behind it. The electrode was then sutured to the dermal layer of the skin flap 


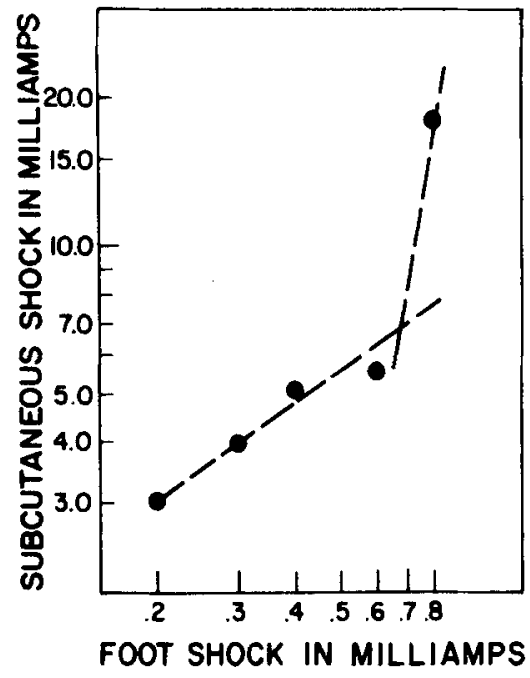

Fig. 1. Equal aversion points for subcutaneous shock and foot shock.

(care being taken not to break through the exterior surface of the skin) with 6-0 surgical silk and the flap sutured back into place with 3-0 silk.

Two pairs of electrode placements were used: (1) $1 / 4$ in. to the right of the midline, approximately bisecting the gluteus maximus muscle, its mate 1 in. further right; and (2) the first of the second pair $1 / 4$ in. to the left of the midline approximately bisecting the acromioctrapezius muscle, its mate $1 \mathrm{in}$. away to the left and slightly caudal behind the pinea of the ear. Next, the $2 \times 2$ strip connector unit was anchored to the head of $S$ by means of five $0-801 / 8$-in. machine screws inserted into holes drilled into the skull (without damaging the meninges) and held in place with dental cement. This assembly was mounted at a forward angle $45 \mathrm{deg}$ to the flat skull. This was done to prevent $S$ from obtaining a firm grip on it and pulling it out.

Out of a total of 96 rats implanted with both tail and neck electrodes or just tail electrodes, only 45 proved suitable for experimental use. The causes of rejection included electrode failure (the wire to the electrode often broke near the base of the implanted connector socket), rejection of the implant (in some Ss the skin overlying the electrode became necrotic and the electrode was then extruded), and other miscellaneous causes, including infection and death.

Experimental design and procedure. The control circuit was arranged so that the rat received a specified "intensity of" subcutaneous shock on one side of the cage and, on the other side, it received shock applied to the feet via the grid floor. For experimental design purposes, footshock was designated the "standard" stimulus and subcutaneous shock, the comparison. At each standard level several comparison intensities were used, the intent being to determine the indifference point for subcutaneous shock vs footshock. Comparison stimuli were chosen on the basis of pilot work to be both more and less aversive than the standard stimuli. The indifference or equal-aversion point was defined as the point where the function relating percent preference to comparison shock intensity crossed the $50 \%$ level. The standard footshock stimulus intensities were $.20, .29, .42, .57$, and $.80 \mathrm{~mA}$. A sixth intensity $(1.14 \mathrm{~mA})$ was used initially, but it required lethal comparison levels of subcutaneous shock and was therefore abandoned.

Six to eight animals were run at each standard with each animal receiving only one intensity of footshock but several comparison intensities. Only one comparison shock was administered during a given test period. The size of the comparison steps was varied from $S$ to $S$ in such a way that 3 to 10 comparison levels per animal per standard were used. The comparisons were ordered either in ascending followed by descending order or vice versa, or with a random order replacing one of the ascending or descending orders.

Each $\mathrm{S}$ was run once or twice daily on weekdays. The side of the cage on which $S$ received grid shock was alternated between trials. If the $S$ had both neck and tail electrodes, the order of their use was counterbalanced. Each trial was $15 \mathrm{~min}$ long.

For each trial $\mathrm{S}$ was removed from its home cage, placed in the preference cage, connected to the commutator, and the circuit checked for continuity. Shock was then turned on and the animal given unescapable shock for the 15-min test period. Time spent on the standard stimulus side was recorded in seconds every $180 \mathrm{sec}$.

In the event that the animal pulled the plug out or failed to cross the cage, he was run again at the same shock level at the next session.

\section{Results}

The mean percent of time spent on the standard side during the last $6 \mathrm{~min}$ of each comparison shock was determined for all standard levels. These percentages were then converted to $Z$ scores, the comparison intensities to logarithms, and a straight line was fitted by the method of least squares. The equal aversion or indifference point for each standard level was defined as the intensity of shock producing equal (50\%) preference for the two shocks, as determined by the fitted function. Figure 1 shows the intensity of subcutaneous shock equal to footshock for all standard levels. The line through the four lower points is the best fitting line, as determined by the least-squares method. The line through the fifth point is arbitrary. These results show that a given intensity of footshock is about 10 times as aversive as an equal intensity (in milliamperes) of subcutaneous shock, except for the highest footshock intensity. At the highest standard level $(.8 \mathrm{~mA})$, proportionally more subcutaneous shock was required to produce equal preference. This change suggests that the high comparison current levels produced tissue damage through electrocauterization or rendered the animal incapable of responding through tetanization of local muscle groups. When high comparison levels were used in an attempt to produce strong preference for $.8-\mathrm{mA}$ shock, several animals were electrocuted or died shortly thereafter.

Other analyses of the data indicated that none of the procedural variations, including number and order of comparison levels, neck vs tail electrodes, or number of trials per day, altered the basic preference function.

\section{EXPERIMENT 2}

The pulse width of the subcutaneous shock used in Experiment 1 resulted in $S$ receiving shock only $67 \%$ of the time vs a constantly varying and indeterminate amount of footshock. This difference in average intensity over time between the two sources may have contributed to the tenfold difference in aversiveness obtained. To examine this possibility, the pulse duration of the subcutaneous shock was varied in the following experiment.

\section{Experimental Design and Procedures}

The apparatus and procedures used in this experiment were identical to those described previously except that (1) only posterior subcutaneous electrodes were used, (2) the pulse forms were changed to include $33 \mathrm{msec}$ on and $167 \mathrm{msec}$ off and continuous shock, and (3) only one standard footshock intensity, $.57 \mathrm{~mA}$, was used.

\section{Results}

The indifference (equal aversion) points for $.57 \mathrm{~mA}$ of footshock were as follows: continuous shock, $10.6 \mathrm{~mA} ; 33 \mathrm{msec}$ on and $167 \mathrm{msec}$ off, $8.2 \mathrm{~mA}$. These two equal aversion points were not significantly different, although the continuous shock was significantly more aversive than the 167-msec-off shock used in the first experiment, where $.57 \mathrm{~mA}$ of footshock was equal in aversiveness to $5.9-\mathrm{mA}$ subcutaneous shock. Taken together these 
equal aversion points suggest that some durations of pulsed shock may be more aversive than continuous shock, but further work is obviously needed to establish this point.

\section{DISCUSSION}

The finding that a given intensity of footshock is $1 / 10$ th as aversive as an equal intensity of subcutaneous shock was both predictable and surprising. At an intuitive level it is easy to view the application of shock to tender unprotected subcutaneous tissue as likely to be excruciatingly painful. Yet receptors in the skin are oriented toward the external surface, suggesting that painful stimulation applied below the skin should not be as effective as one at its surface. Another factor that may contribute to the painfulness of footshock is that as the animal moves about on the grid he makes and breaks contact with the electrodes, causing variations in current density, which may increase painfulness of the stimulus (Campbell \& Masterson, 1969).

The change in slope of the equal aversion function between .57 and $.80 \mathrm{~mA}$ of footshock probably reflects tissue-damaging electrocautery, receptor-cell hyperpolarization, and/or muscular tetanization, conditions that either reduce or make the animal unable to respond to painful stimulation. What is particulary striking about these intense, sometimes lethal, levels of subcutaneous shock is that they are less aversive, as judged by preference, than intensities of footshock far below a physically damaging level.

In view of the lengthy surgery, expense, poor recovery rate, and high amounts of current needed, subcutaneous shock is clearly not suitable for general use. It may, however, be useful for special purposes, such as comparing spatial and temporal interaction of painful stimuli (cf. Messing \& Campbell, in press).

\section{REFERENCES}

APPEL, J. B. Drugs and escape behavior. Report on Research Grant MH 07239 submitted to National Institute of Mental Health, 1965.

BARFIELD, R. J., \& SACHS, B. J. Sexual behavior: Stimulation by painful electrical shock to skin in male rats. Science, 1968, 161, 392-395.

CAMPBELL, B. A., \& MASTERSON, F. A Psychophysics of punishment. In B. A. Campbell and R. M. Church (Eds.)
Punishment and aversive behovior. New York: Appleton-Century-Crofts, 1969. Pp. 3-42.

HAKE, D. F., \& AZRIN, N. H. An apparatus for delivering pain-shock to monkeys. Journal of the Experimental Analysis of Behavior, 1963, 6, 297.

HUTCHINSON, R. R., AZRIN, N. H., \& HAKE, D. F. An automatic method for the study of aggression in squirrel monkeys. Joumal of the Experimental Analysis of Behavior, 1966, 9. 233-237.

KELLY, N. D., \& GLUSMAN, M. Aversive thresholds for subcutaneous electrical stimulation in the cat. Psychonomic Science, 1964, 1, 207-208.

MESSING, R. B., \& CAMPBELL, B. A. Summation of pain produced in different anatomical regions. Perception \& Psychophysics, in press.

VALENSTEIN, E. S. A note on anesthetizing rat and guinea pigs. Journal of the Experimental Analysis of Behavior, 1961, 4, 6 .

WEISS, J. A tail electrode for restrained rats, Journal of the Experimental Analysis of Behavior, 1967, 10, 85-86.

\section{NOTES}

1. This research was supported in part by NSF Grant GB 8046 .

2. Requests for reprints should be sent to Byron A. Campbell, Department of Psychology, Green Hall, Princeton University, Princeton, N.J. 08540 .

3. Now at Nebraska Psychiatric Institute, Nebraska College of Medicine, Omaha, Nebraska 68105 .

\section{A constant-current shock source for providing direct or alternating current output ${ }^{1}$}

ROBERT A. STEWART ${ }^{2}$ and SAM L CAMPBELL ${ }^{3}$ CALIFORNIA STATE COLLEGE AT LOS ANGELES, College Drive, Los Angeles, California 90032

A vacuum-tube constant-current shock source, providing ac, smooth $d c$, and pulsating dc output is described. In addition to output flexibility, the shock source is compact, inexpensive to construct, and has undergone 4 years of testing and laboratory use. Operating instructions, theory of operation, and suggested modifications are included along with performance specifications.

Electric shock is the most frequently used noxious stimulus in psychological research, but it is also one of the least standardized experimental variables. Ss have been shocked with smooth $\mathrm{dc}$, pulsating dc, mono- and biphasic square waves, and currents with irregular waveforms. Because of this plethora of shocking currents, the three-mode shock source described in this paper was developed.
The shock source provides three constant current outputs: $60-\mathrm{Hz}$ ac, $120-\mathrm{Hz}$ pulsating $\mathrm{dc}$, and smooth dc. The term constant current, when applied to constantly changing waveforms (ac and pulsating dc), means generally that successive cycles have identical average and instantaneous current values. Other design aims were high circuit efficiency and simplicity, which contribute materially to reliability, compactness, and low cost. The three-mode shock source requires only 19 electronic components and costs less than $\$ 40$, including a current monitoring meter.

\section{PERFORMANCE SPECIFICATIONS}

The performance of the shock source is a function of the mode of operation, the level of output current, and the resistance of the S. Except for waveform, the electrical specifications of the ac and pulsating dc outputs are identical. In these modes, an increase in load resistance ( $S$ resistance) from 0 to $50,000 \mathrm{ohms}$ results in the following decrements in output current: $1.0 \%$ at $1 \mathrm{~mA}, 1.5 \%$ at $2 \mathrm{~mA}$, $2.0 \%$ at $3 \mathrm{~mA}, 5.0 \%$ at $4 \mathrm{~mA}, 12.0 \%$ at
$5 \mathrm{~mA}$, and $19.0 \%$ at $6 \mathrm{~mA}$. The smooth dc mode is the most efficient. The filter capacitors charge to the peak voltage of the power transformers, and the peak current is equal to the average output current. In the smooth dc mode, a 50,000-ohm increase in $\mathrm{S}$ resistance causes the following current decrements: $0.5 \%$ at $1 \mathrm{~mA}, 1.0 \%$ at $2.3 \mathrm{~mA}, 2.0 \%$ at $4 \mathrm{~mA}, 4.0 \%$ at $5 \mathrm{~mA}$, and $6.0 \%$ at $6 \mathrm{~mA}$.

The output voltage of a constant-current shock source is a function of the selected current level and the resistance of a given S. However, the open-circuit (no load) output voltage is known and allows, by Ohm's law, the calculation of the maximum $S$ resistance through which a desired current can be maintained. The effective voltage (RMS) for the ac and pulsating dc modes is $250 \mathrm{~V}$; the open-circuit output voltage in the smooth dc mode is $350 \mathrm{~V}$. The latter voltage exceeds the design-maximum plate voltage of the 6AU6 vacuum tube (RCA, 1965), but in the past 4 years no tube has failed catastrophically, nor has tube life been short. 